\title{
The effect of Nd:YAG laser therapy on cutaneous leishmaniasis compared to intralesional meglumine antimoniate
}

\author{
Mohammad Omidian' ${ }^{1}$, Maryam Jadbabaei², Ehsan Omidian³ ${ }^{3}$ Zahra Omidian ${ }^{4}$
}

\begin{abstract}
${ }^{1}$ Department of Dermatology, Ahvaz Jundishapur University of Medical Sciences, Ahvaz, Iran ${ }^{2}$ Student Research Committee, Ahvaz Jundishapur University of Medical Sciences, Ahvaz, Iran ${ }^{3}$ Private Clinic of Dermatology, Ahvaz, Iran

${ }^{4}$ Department of Pathology, Johns Hopkins University School of Medicine, Baltimore, USA
\end{abstract}

Adv Dermatol Allergol 2019; XXXVI (2): 227-231

DOI: https://doi.org/10.5114/ada.2019.82827

\begin{abstract}
Introduction: Nd:YAG laser is one of the commonly used lasers in dermatology.

Aim: This study aimed to investigate the effect of Nd:YAG laser therapy (NDY) on cutaneous leishmaniasis in comparison with meglumine antimoniate (MA). Therefore, researchers are seeking to use a more effective, faster and less complicated method.

Material and methods: This study was conducted as a clinical trial on 16 patients with cutaneous leishmaniasis treated simultaneously as follows: one lesion with Glucantime and another with NDY laser in the Ahvaz Jundishapur University of Medical Sciences in 2016-2017. Then, the demographic data, number of treatment sessions, mean duration of illness before the start of treatment, post inflammatory hyperpigmentation (PIH), and scars, and recovery in two methods were recorded and compared using SPSS-21.

Results: The mean age was $14.37 \pm 29.68$. The mean duration of disease before the study was $1.84 \pm 0.50$ months. The mean number of Glucantime injections was $7.31 \pm 4.01$ and the mean number of laser therapy sessions was $2.56 \pm 0.89$; this was significantly less than that of injections of the MA group $(p<0.001)$. Moreover, the MA scars were observed in 10 subjects and laser scars were seen in 3 subjects. Scars in patients treated with laser were significantly smaller than in those treated with Glucantime injections $(p=0.03) .13$ subjects had MA therapy-induced $\mathrm{PIH}$ and 15 had laser therapy-induced PIH.

Conclusions: The alternative method of laser use in the treatment of patients with cutaneous leishmaniasis can lead to complete recovery of patients in shorter time and with less complications.
\end{abstract}

Key words: cutaneous leishmaniasis, meglumine antimoniate, $\mathrm{Nd}$ :YAG laser.

\section{Introduction}

Leishmaniasis is caused by Leishmania species, a type of protozoan of the threanosomatoid family, and is a mandatory cell transmitted through the mammals-infected bite of the sand flies [1]. According to the definition of the World Health Organization, Leishmaniasis is divided into the new world and the old world [2]. Cutaneous leishmaniasis is endemic in 88 countries (Asia, Latin America, Africa and southern Europe). Three hundred fifty million people are at risk of this disease, 12 million suffer from it and 1.5 million people are affected each year. Cutaneous leishmaniasis occurs in central and southwestern Asia, in both dry (urban) and wet (rural) forms. The most important vector of the Leishmania Major in Iran is the sand flies of the group of Caucasicus (Phlebotomus papatasi) and Alexander [3]. Rural leishmaniasis is mostly seen in the southwest of Iran and the notably among the rodents. Classical clinical manifestations of cutaneous leishmaniasis are seen as erythematous nodules and papules at the site of the insect bite, which develops into a lesion and will heal after a few months to several years, rare variants are erysipeloid, annular, paranochial, palmoplantar, sporotrichoid and zosteriform [4-6]. There are several types of systemic and topical treatments for cutaneous leish-

Address for correspondence: Dr. Maryam Jadbabaei, Student Research Committee, Ahvaz Jundishapur University of Medical Sciences, Ahvaz, Iran, phone: +98 9161110983, e-mail: drjadbabai.maryam@gmail.com

Received: 9.12.2017, accepted: 10.12.2017. 
maniasis. Systemic types include pentavalent antimony compounds (Glucantime) and oral medicines (fluconazole, zinc sulfate and azithromycin). Topical treatments include intralesional injections of pentavalent compounds, topical drugs (Imiquimod and Paromomycin) and physical methods (cryotherapy, laser $\mathrm{CO}_{2}$, the local heat, photodynamic therapy and surgical excision of the lesion) [7, 8]. Pentavalent antimony compounds such as Glucantime (Meglumine antimoniate) are the first-line treatment of leishmaniasis. This drug, despite its positive effect on the treatment process, has some disadvantages. Complications of Glucantime include elevated liver enzymes, electrocardiographic changes, skin rash, and painful injection [9]. Moreover, this drug should be used for several days, while some cases of resistance to glucantime have been reported in Iran [10,11]. Neodymium-Doped Yttrium Aluminum Garnet (Nd:YAG) is one of the commonly used lasers in dermatology. Its wavelength is $1064 \mathrm{~nm}$, which has the highest penetration depth among the different types of lasers and hemoglobin was its main chromophore.

\section{Aim}

Since intralesional injection of Glucantime is very painful, requires weekly injections and may be associated with systemic complications, and there may be resistance to glucantime, the aim of this study is to investigate the effect of Nd:YAG (NDY) laser therapy on cutaneous leishmaniasis compared to Glucantime [12, 13].

\section{Material and methods}

After obtaining permission from the Ethics Committee of Ahvaz Jundishapur University of Medical Sciences, this clinical trial was conducted as a pilot study for the treatment of leishmaniasis in 2016-2017. Samples were selected from among patients with cutaneous leishmaniasis referring to the 17 Shahrivar Health Treatment Center of Ahvaz.
The inclusion criteria included age: 5-60 years old, having at least two lesions of C. Leishmaniasis diagnosed by 2 dermatologists and with a positive test of direct smear in terms of Leishman body, confirmed by a pathologist. Exclusion criteria include pregnancy or breastfeeding, any previous treatment including medical treatment, cryotherapy or laser therapy or other destructive methods.

Before the start of treatment, written consent was received from all patients or their parents. In the first session, two distinct C. Leishmaniasis lesions were randomly determined on the patient's body. It was first marked by a marker and then, photographed with a 16-megapixel Canon camera. Afterwards, each of the two lesions of the patient was treated by one of the two following treatments:

- 1500 mg/5 $\mu \mathrm{l}$ Meglumine Antimoniate was administered by intradermal injection into one of the lesions. Maximum drug injected per session was $1-2 \mu \mathrm{l}$. Weekly injections continue until complete recovery of the lesion.

- Another lesion was treated with Nd:YAG laser with the specifications of the Clarity Lutronic laser and the Fluence: $200 \mathrm{mj} / \mathrm{cm}^{2}$, Pulse duration: $20 \mathrm{~ms}$, Spot Size: $3 \mathrm{~mm}$ indexes.

Treatment with Nd:YAG laser was repeated at intervals of two weeks until complete recovery of the lesion (lack of induration) and direct smear from lesions becomes negative.

In each treatment session, a patient's lesion was photographed again. Follow-up of skin lesions was conducted after the healing and three months later. In each session, the lesion was examined in terms of the scar and Post Inflammatory Hyperpigmentation (PIH) and was photographed. At the end, collected data were analyzed by SPSS-21 and the results were recorded.

\section{Results}

Table 1 shows the results of our study. The results showed that the mean age of the subjects was $29.7 \pm 14.4$

Table 1. The comparison between Glucantime-based treatment and Nd:YAG laser treatment methods

\begin{tabular}{|c|c|c|c|c|}
\hline Parameter & & Mean & SD & $P$-value \\
\hline \multirow[t]{2}{*}{ Age [year] } & Male & 26.4 & 15.43 & $<0.001$ \\
\hline & Female & 32.2 & 13.9 & \\
\hline \multirow[t]{2}{*}{ Number of sessions } & Glucantime & 7.3 & 4.0 & \\
\hline & YAG laser & 2.6 & 0.9 & \\
\hline Parameter & & Number $(N=16)$ & Percent (100) & $P$-value \\
\hline \multirow[t]{2}{*}{ Scars } & Glucantime & 10 & 62.5 & 0.03 \\
\hline & YAG laser & 3 & 18.7 & \\
\hline \multirow[t]{2}{*}{$\mathrm{PIH}$} & Glucantime & 13 & 81.3 & $>0.05$ \\
\hline & YAG laser & 15 & 93.8 & \\
\hline \multirow[t]{2}{*}{ Improvement } & Glucantime & 16 & 100.0 & $>0.05$ \\
\hline & YAG laser & 16 & 100.0 & \\
\hline
\end{tabular}


years old. The mean age of the male patients was 26.4 \pm 15.4 and the mean age of the female patients was 32.2 \pm 13.9 years old. There were seven men and nine women. The mean duration of the disease before the study was $1.8 \pm 0.5$ months. The number of treatment sessions showed that the mean number of Glucantime injections was $7.3 \pm 4.0$ and the mean number of laser sessions was $2.6 \pm 0.9$. Also, in terms of scar frequency, it was observed that in the Glucantime-based treatment, 10 patients had scars but six ones did not have any. In the laser-based treatment method, three patients had a scar, but 10 had no scar, also, three patients had hypertrophic scar complications. The PIH frequency for 16 patients also indicated that 13 people had Glucantime-induced PIH but three had no PIH. And 15 from 16 patients had laser therapyinduced $\mathrm{PIH}$, but only one had no PIH.

The results of the two therapeutic methods were compared and different research variables were analyzed. It was reported that post-treatment recovery was positive in all 16 patients in both laser and Glucantime injections and the recovery of both methods was equal. For determining the relationship between sex and post-treatment recovery, Fisher's exact test was used. There was no significant dif-
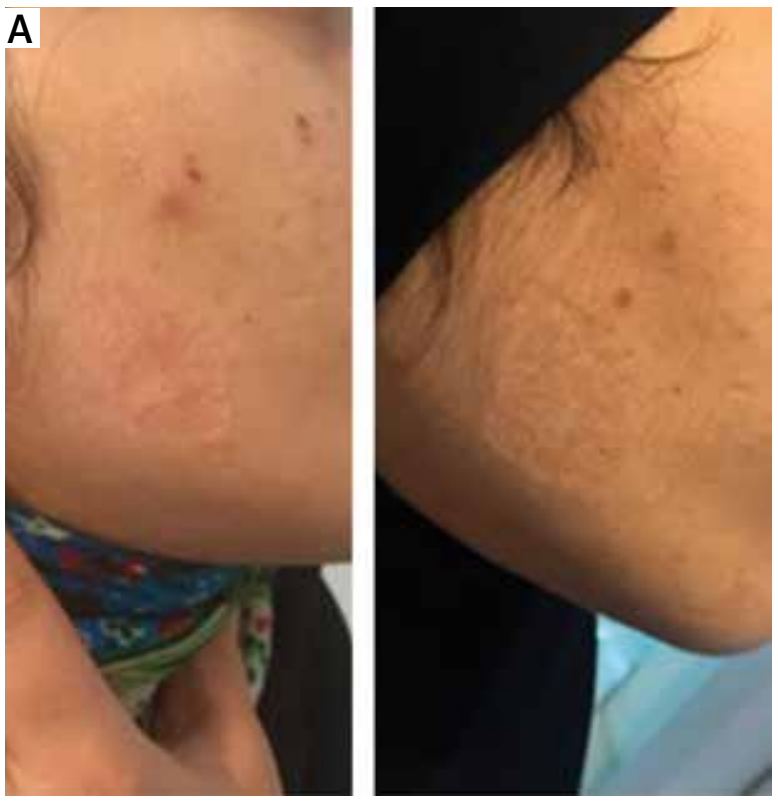

Figure $1 \mathrm{~A}-\mathrm{C}$. Lesions of cutaneous leishmaniasis before and after Nd:YAG laser treatment ference between the two groups. In order to determine the relationship between PIH and sex of the patients, Fisher's exact test was used. There was no statistically significant difference. Fisher's exact test was used to determine the relationship between sex and scar. No significant statistical relationship was found. As previously stated, in the Glucantime-based method, there were 10 patients with scars, 6 males and 4 females, and in the laser-based method, there were three patients with scars, all of whom were females. In order to compare the mean number of treatment sessions and sex, Mann-Whitney non-parametric test was used. There was no significant relationship between them. As mentioned before, the mean number of Glucantime injections was $4.0 \pm 7.3$, and the mean number of laser sessions was $2.6 \pm 0.9$.

To determine the relationship between the duration of the disease and the rate, for Nd:YAG laser therapy and Glucantime method, Mann-Whitney non-parametric test was used, but since the recovery was observed in all patients, no significant level was reported. Besides, in order to compare the mean duration of the disease and PIH, Mann-Whitney non-parametric test was used, and the results were not statistically significant. In order to compare the mean score of
B

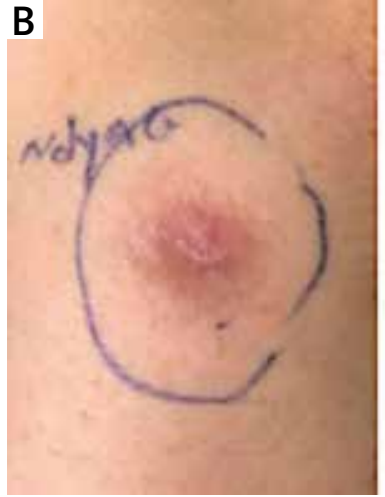

C

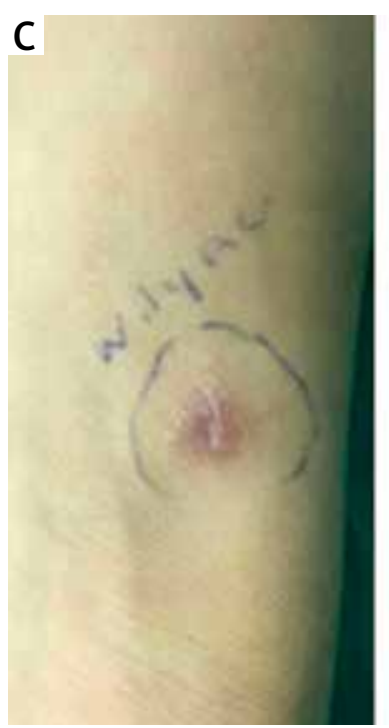

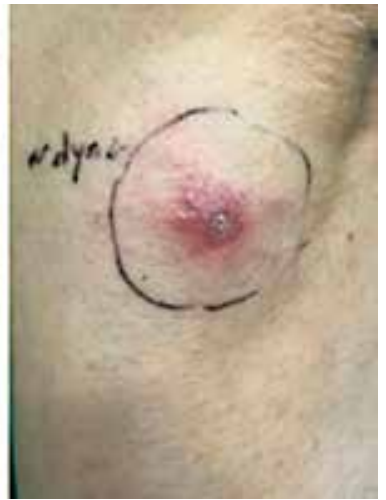

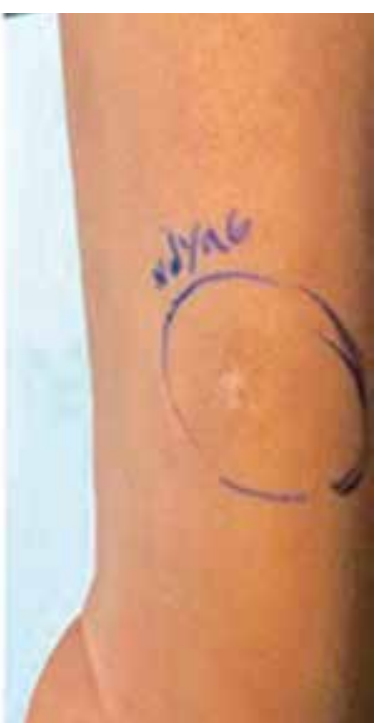


disease duration and scars, Mann-Whitney non-parametric test was used, but no significant difference was found.

Fisher's exact test was also used to determine the relationship between the type of treatment and the rate of recovery. However, since both treatments were positive for all patients, there was no difference between the two methods $(p>0.05)$. To determine the relationship between the type of treatment and PIH, Fisher's exact test was used and there was no significant difference $(p>0.05)$.

However, in order to investigate the relationship between the type of treatment and the scar, Fisher's exact test was used. Results revealed that patients treated with laser had significantly less scars than those treated with Glucantime $(p=0.03)$. Furthermore, in order to investigate the relationship between the type of treatment and the number of treatment sessions, Mann-Whitney test was used. Results showed that the number of treatment sessions in the laser-based method was significantly lower than in the Glucantime-based method ( $p<$ 0.001). Figure 1 shows the lesions of cutaneous leishmaniasis in our patients before and after YAG laser treatment.

\section{Discussion}

The results show that a higher number of women (56.3\%) participated in this study than men and that the mean duration of the disease was $0.5 \pm 1.8$ months. Among the two treatments, the use of Glucantime at an interval of 2 to 15 sessions resulted in recovery, however, the laser method resulted in recovery only in the 1-4 session interval. Therefore, one of the significant results of this study was reduction in the number of treatment sessions in the laser-based method, indicating that the use of laser significantly reduces the number of sessions. Also, the frequency of the scar was another significant variable in the present study as in the Glucantime-based method, $62.5 \%$ of patients had a scar, however, in the laser-based method, only $18.7 \%$ had scars, which was significantly lower. Of course, three patients had a laserinduced hypertrophy scar, which was the only complication recorded in this study. There was no significant difference in $\mathrm{PIH}$ between the two methods, however, $\mathrm{PIH}$ was $81.3 \%$ for Glucantime-based method and $93.8 \%$ for the laser-based method. However, the type of laser used in other studies is different from the present study. Radmanesh and Omidian studied pulsed dye laser (PDL) for treatment of 17 patients with 81 lesions of cutaneous leishmaniasis [14]. They treated 42 lesions with one or two passes of PDL fortnightly and 39 lesions in the same patients with intralesional meglumine antimoniate (MA) weekly. They showed that PDL cured many cases of cutaneous leishmaniasis faster than MA particularly in the early stages. In one of the relatively old studies that Babajev et al. conducted on 108 patients, they used a $\mathrm{CO}_{2}$ laser to treat Leishmaniasis lesions and concluded that all patients had recovered after 22 days on average. In this study, no recurrence was observed after a 7-year follow-up period [15], although in this study, the number of treatment sessions was higher: more than 5 times than that of the present study, but the recovery of the patients was consistent with this study. In another study, Asilian et al. carried out a clinical trial in Isfahan, Iran in 2004 and treated 123 patients with cutaneous leishmaniasis with a total of 183 leishmaniasis lesions using $\mathrm{CO}_{2}$ laser. They also treated another group of 110 patients (with a total of 250 leishmaniasis lesions) simultaneously with Glucantime and followed up all patients for relapse and treatment complications at weeks $1,3,4,8,12$, and 24 after treatment. The results of this study showed that $\mathrm{CO}_{2}$ laser was more effective than Glucantime in the treatment of leishmaniasis, and in the laser therapy group there were less complications of the treatment and they were limited to the lesion site [16]. The results of this study were also consistent with our study, and the important point is that they only recovered after a single treatment session, and this finding needs further investigation with respect to the number of longer sessions of treatment in the Babajev's study that used a similar laser. In other studies, similar results have been observed with less recovery, including Asilian in 2006 reported the results of laser $\mathrm{CO}_{2}$ treatment on 24 patients with Lupoid cutaneous leishmaniasis. The effect of the laser was determined based on the recovery and non-recurrence within 1 year of treatment. Laser therapy in these patients was well tolerated and minimal complications were reported including pain, dry skin, and mild erythema. In this study, 19 patients completely recovered and only one failure was reported [17]. But among other studies, the use of other different lasers has also been observed. For example, Al Muslet and Khalid in 2004 in Sudan performed a study on 13 patients with cutaneous leishmaniasis treated with a diode laser. They used a diode laser with a wavelength of $820 \mathrm{~nm}$ three times a week, 10 sessions in total. The effect of the laser therapy in this study was defined as reducing inflammation and accelerating wound healing. The reported results showed an excellent response in $92 \%$ of treated patients. Complications were small and temporary [18]. In their study, although the results were consistent, the recovery rate was lower than in the present study, and the number of treatment sessions was higher, indicating that the laser was more effective than that in the present study. Meymandi et al. in 2011 used a $\mathrm{CO}_{2}$ laser session to treat cutaneous leishmaniasis in 96 patients in a controlled study in Kerman, Iran. They also treated another group of 95 patients with intralesional injections of the Meglumine Antimonite and cryotherapy for 12 weeks. The results of the treatment and its complications were evaluated in the two groups, and all patients were followed up at weeks 2, 6, 12 and 16 after treatment. At the end of the study, it was observed that complete recovery was achieved in $93.7 \%$ of the patients treated with laser 
and $78 \%$ of patients treated with medication and cryotherapy. Complications were similar in both groups and limited to the site of the wound [19]. This finding, which is spatially and temporally closer to the present study, again reveals that the laser used in the present study was more effective than the $\mathrm{CO}_{2}$ laser.

\section{Conclusions}

In general, the results of this study and the review of other studies demonstrate that the alternative method of laser therapy for the treatment of patients with cutaneous leishmaniasis can lead to a complete recovery of patients in a shorter time with less complications. Indeed, it is suggested that a similar study should be done with a larger sample size, as well as follow-up of patients over a longer period of time in order to achieve better results of using YAG laser.

\section{Conflict of interest}

The authors declare no conflict of interest.

\section{References}

1. Garcia LS. Diagnostic Medical Parasitology. $5^{\text {th }}$ edn. Washington DC: American Society for Microbiology Press 2007.

2. Murray HW, Berman JD, Davies CR, Saravia NG. Advances in leishmaniasis. Lancet 2005; 366: 1561-77.

3. Randazzo S, Guarneri B, Messina V, Palazzo R. Leishmaniosi Cutanea Mediterranea. Cilag, Schaffausen 1990; 6-45.

4. Samady JA, Schwartz RA. Old World cutaneous leishmaniasis. Int J Dermatol 1997; 36: 161-6.

5. Willard RJ, Jeffcoat AM, Benson PM, Walsh DS. Cutaneous leishmaniasis in soldiers from Fort Campbell, Kentucky returning from Operation Iraqi Freedom highlights diagnostic and therapeutic options. J Am Acad Dermatol 2005; 52: 977-87.

6. Omidian M, Mapar MA. Chronic zosteriform cutaneous leishmaniasis. Indian J Dermatol 2006; 72: 41-2.

7. Minodier P, Parola P. Cutaneous leishmaniasis treatment. Travel Med Infect Dis 2007; 5: 150-8.

8. Nilforoushzadeh MA, Shirani-Bidabadi L, Zolfaghari-Baghbaderani A, et al. Topical effectiveness of different concentrations of nanosilver solution on Leishmania major lesions in Balb/c mice. J Vector Borne Dis 2012; 49: 249-53.

9. Hajjaran H, Mohebali M, Razavi MR, et al. Identification of Leishmania species isolated from human cutaneous leishmaniasis, using random amplified polymorphic DNA (RAPDPCR). Iranian J Publ Health 2004; 33: 8-15.

10. Zachary CB, Rofagha R. Laser therapy. Dermatology. $3^{\text {rd }}$ edn. London, Mosby 2012.

11. Baptista C, Miranda LD, Madeira MD, et al. In vitro sensitivity of paired Leishmania (viannia) braziliensis samples isolated before meglumine antimoniate treatment and after treatment failure or reactivation of cutaneous leishmaniasis. Dis Markers 2015; 2015: 943236.

12. Kawashima Y, Aoki A, Ishii S, et al. Er:YAG laser treatment of gingival melanin pigmentation. International Congress Series 2003 May 31 (Vol. 1248, pp. 245-248).
13. Tal H, Oelgiessr D, Tal M. Gingival depigmentation for aesthetic purposes using erbium:YAG laser: rationale and technique. Refu'at ha-peh veha-shinayim (1993) 2002; 19: 25-32.

14. Radmanesh M, Omidian E. The pulsed dye laser is more effective and rapidly acting than intralesional meglumine antimoniate therapy for cutaneous leishmaniasis. J Dermatol Treat 2017; 28: 422-5.

15. Babajev KB, Babajev OG, Korepanov VI. Treatment of cutaneous leishmaniasis using a carbon dioxide laser. Bull World Health Organ 1991; 69: 103-6.

16. Asilian A, Sharif A, Faghihi G, et al. Evaluation of $\mathrm{CO}_{2}$ laser efficacy in the treatment of cutaneous leishmaniasis. Int J Dermatol 2004; 43: 736-8.

17. Asilian A, Iraji F, Hedaiti HR, et al. Carbon dioxide laser for the treatment of lupoid cutaneous leishmaniasis (LCL): a case series of 24 patients. Dermatol Online J 2006; 12: 3 .

18. Al-Muslet NA, Khalid Al. Clinical evaluation of low level laser therapy in treatment of cutaneous leishmaniasis. Our Dermatol Online/Nasza Dermatologia Online 2012; 3: 196-201.

19. Meymandi S, Zandi S, Aghaie H, Heshmatkhah A. Efficacy of CO2 laser for treatment of anthroponotic cutaneous leishmaniasis, compared with combination of cryotherapy and intralesional meglumine antimoniate. J Eur Acad Dermatol Venereol 2011; 25: 587-91. 